
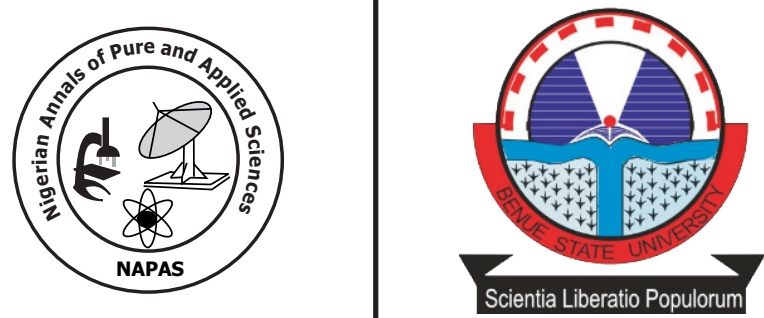

\title{
Digital Image Segmentation Using the Delaunay Triangulation Algorithm
}

\author{
Onoja, G.U., and Aboiyar,T. \\ Department of Mathematics/Statistics/Computer Science, \\ University of Agriculture, Makurdi, Benue State, Nigeria. \\ onojason2003@yahoo.com,t_aboiyar@yahoo.co.uk
}

\section{Abstract}

Image segmentation is the process of partitioning a digital image into multiple segments according to some homogeneity criterion. Different approaches are suited to different types of images and the quality of output of a particular algorithm is difficult to measure quantitatively due to the fact that there may be much "correct" segmentation for a single image. Although the field encounters several challenges, this research work seeks to present a segmentation algorithm for gray intensity images. Image features extraction is first performed to obtain the approximate, if not exact, representation of a given image. Image enhancement was also carried out by exploiting the principle of histogram equalization which is one of the well-known image enhancement technique. The proposed approach is based on the Delaunay (DT), one of the techniques in computational geometry, which generates clusters of intensity values using information from the vertices of the external boundary of triangulation. This makes it possible to produce segmented image regions. The algorithm was tested on sampled images before and after enhancement and the production compared. Our algorithm successfully extracts cogent features from a given image and made use of the Delaunay Triangulation to carry out segmentation. The method is robust, precise and independent of translation, rotation and scaling and that makes the result quite good for image segmentation. Image segmentation based on Delaunay triangulation technique seem to be a promising future research area with respect to its applicability to underwater mine and submarine detection, better target recognition, medical imaging as well as to automated computer vision.

Keywords: Delaunay Triangulation, Digital Image, Image segmentation, Image enhancement, Histogram equalization 


\section{Introduction}

Mesh generation, defined as the process of breaking up a physical domain into smaller sub-domains (elements), is a critical step in a wide range of applications including scientific computing, computer graphics, surveying, terrain databases and in the numerical solution of partial differential equations.

Mesh generation is a topic studied by two kinds of researchers; theoreticians (mainly mathematics and computer scientists), and practitioners (mainly structural engineers). The theoretical study of mesh generation is part of the field of computational geometry, while the practical study is noted in computer simulation of physical problems. Whereas engineers are satisfied with mesh generators that usually work for their chosen domains, computational geometers set a loftier goal; provably good mesh generation, the design of algorithm that are mathematically guaranteed to produce a satisfying mesh [9].

One of the most popular meshing algorithms is the Delaunay triangulation method which starts from an initial triangulation and refines until the element qualities are sufficiently high. Since its invention in 1934, Delaunay triangulation has been used in various fields of computer graphics and computational geometry. Examples of its use include terrain modelling, building meshes for the finite element method and many others. Pertinent to mention is image segmentation which is one of the most important precursors for image processing-based applications and has a crucial impact on the overall performance of the developed systems because every segmentation process consists of dividing the input image into several disjoint regions with similar characteristics such as colour and texture.

Recently, the task of partitioning an image into non-intersecting regions such that each region is homogeneous and the union of no two adjacent regions is homogeneous has attracted various researchers into this very area of computer science. Segmentation is an inherently subjective problem and so several segmentation algorithms have been proposed and implemented with each having its uniqueness. However, there is still not an exact solution that can be applied to all image types and obtains perfect results. Robust segmentation has been the subject of research for many years, but till now published works indicate that most of the developed image segmentation algorithms have been designed in conjunction with particular applications. It is a difficult task since often the scene objects are defined by image regions with nonhomogenous texture and colour characteristics and in order to divide the input image into semantically meaningful regions many developed algorithms either use a priori knowledge in regard to the scene objects or employ the parameter estimation for local texture [7]. There is still no robust solution to the image segmentation problem. There are two main reasons, the first is that the content variety of images is too large, and the second one is that there is no benchmark standard to judge the performance [21]. The goal of segmentation is typically to locate certain objects of interest which may be depicted in the image.

In this work, we will use the Delaunay triangulation to segment digital images and extract features. Given an digital image, we would partition the image into groups of pixels which are homogeneous with respect to some criterion. Different groups must not intersect each other and adjacent groups must be heterogeneous. The Image will first of all be enhanced by histogram equalization and the segmentation process will be carried out iteratively. This would be by using Delaunay triangulation of the image histogram. This is an extension of the work of [4].

\section{Triangulations \\ Delaunay Triangulation}

Delaunay triangulation and the related construction methods resulting in this triangulation have been extensive fields of research for a very long time. In particular, these topics are one of the major concerns in computational geometry. There is a great deal of literature about Delaunay triangulation, starting with the pioneering paper by Delaunay himself, [6]. Delaunay triangulation problems are of interest for a number of reasons. Firstly, numerous theoretical issues can be investigated. Then, a wide range of applications in various disciplines exists, including geometry, computational geometry, as well as computer science and many engineering problems. Triangulation methods are important ingredients in finite element methods (FEM), numerical approximation, computer-aided geometric design (CAGD), and various other 
application fields. They form a part of the wholesome mesh generation methods widely used today in very many areas of scientific and technological research and development. The choice of the mesh type is clearly related to the application [23].

A triangulation of a discrete point set $\mathrm{X}$ $=\left\{\mathrm{x}_{1}, \ldots, \mathrm{x}_{\mathrm{N}}\right\}$ is a collection $\mathrm{T}_{\mathrm{x}}=\{\mathrm{T}\}_{\mathrm{T} \in \mathrm{TX}}$ of triangles in the plane, such that the following conditions hold.
(a) The vertex set of $T_{x}$ is $X$;
(b) Any pair of two distinct triangles in $T_{x}$ intersect at most at one common vertex or along one common edge;
(c) The convex hull $[\mathrm{X}]$ of $\mathrm{X}$ coincides with the area covered by the union of the triangles in $\mathrm{T}_{\mathrm{x}}$;

We say that a collection $\mathrm{T}=\{\mathrm{T}\}_{\mathrm{T} \in \mathrm{T}}$ of triangles is a triangulation of a connected set $\Omega \subset \mathrm{R}^{2}$ iff property (b) holds and $\Omega$ is the union of the triangles in $\mathrm{T}$.

Furthermore, the Delaunay triangulation $\mathrm{D}_{\mathrm{x}}$ of a discrete planar point set $\mathrm{X}$ is a triangulation of $\mathrm{X}$, such that the circumcircle for each of its triangles does not contain any point from $\mathrm{X}$ in its interior. We recall three important properties of Delaunay triangulations.

(a) The Delaunay triangulation $\mathrm{D}_{x}$ of $\mathrm{X}$ is unique, provided that no four points in $\mathrm{X}$ are co-circular. We remark that there are efficient computational methods for choosing, on given $\mathrm{X}$ a Delaunay triangulation of $\mathrm{T}$ without ambiguity. Therefore, we will from now assume that $D_{x}$ is the unique Delaunay triangulation of $\mathrm{X}$.

(b) On given point set $\mathrm{X}$ of size $\mathrm{N}=|\mathbf{x}|$, its (unique) Delaunay triangulation $\mathrm{D}_{\mathrm{x}}$ can be computed in $\mathrm{O}(\mathrm{N} \log (\mathrm{N}))$ steps.

(c) For any Delaunay triangulation $\mathrm{D}_{\mathrm{x}}$ of a point set $\mathrm{X}$, its dual graph is the Voronoi diagram V of X.

In accordance with the above definition, a triangulation can also be regarded as a planar graph, whose nodes are the vertices of the triangles, and whose edges provide the connectivity in the graph [18]. Thus, the basic entities in a triangulation are triangles, edges, and nodes.

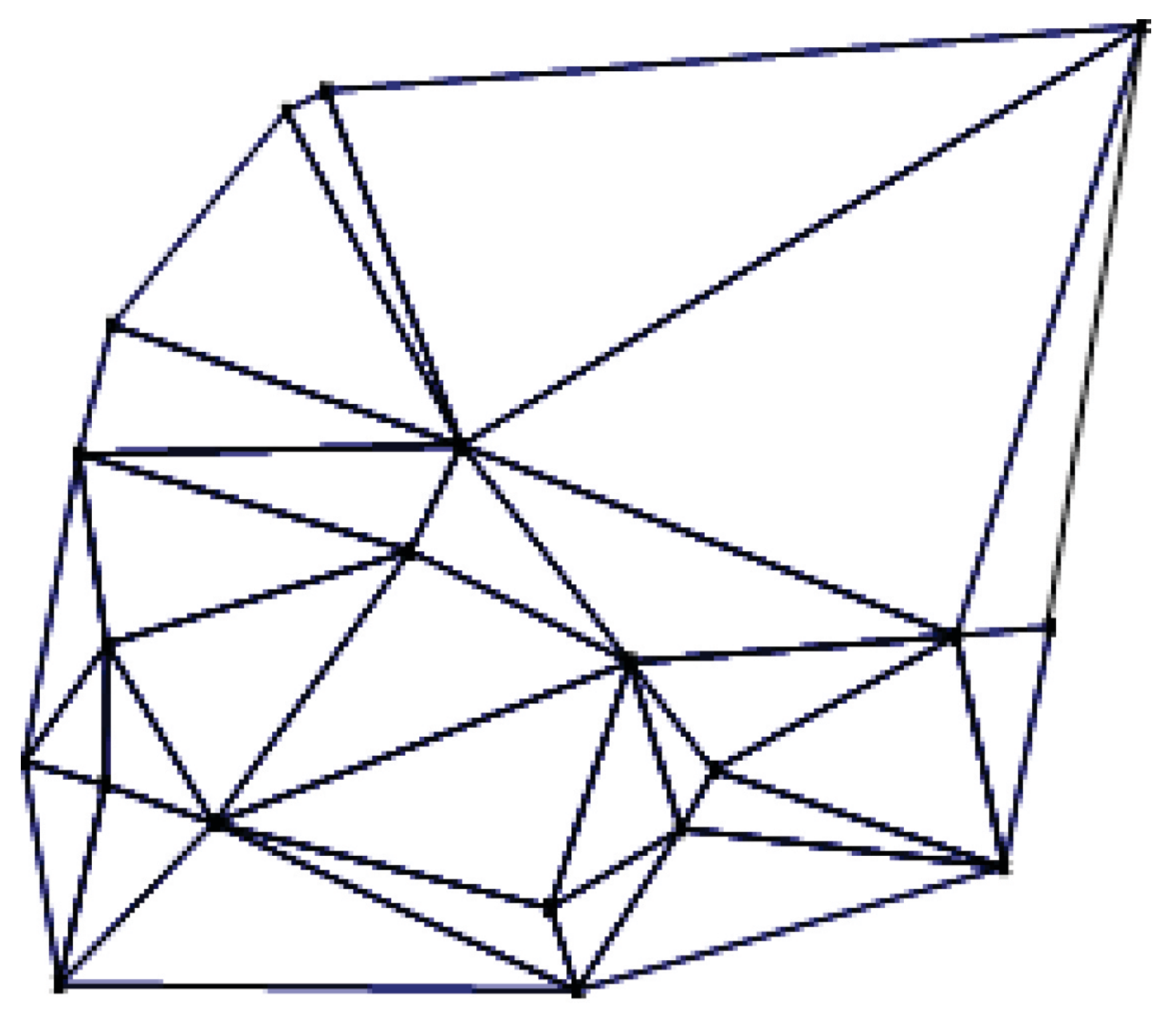

Figure 1: A Triangulation of a Point Set X of Size 
By the classical Euler formula, we have, for any triangulation $T$, the relationship $\mathrm{N}_{\mathrm{v}}-\mathrm{N}_{\mathrm{e}}$ $+\mathrm{N}_{\mathrm{t}}=1$

For its number $\mathrm{N}_{\mathrm{t}}$ of triangles, $\mathrm{N}_{\mathrm{e}}$ of edges, and $\mathrm{N}=$ Nvof vertices. Moreover, with letting $\mathrm{N}^{\mathrm{b}}$ the number of boundary points in $\mathrm{T}$, we obtain the formulae

$\mathrm{N}_{\mathrm{t}}=2 \mathrm{~N}-\mathrm{N}^{\mathrm{b}}-2$

$\mathrm{N}_{\mathrm{e}}=3 \mathrm{~N}-\mathrm{N}^{\mathrm{b}}-3$

For any Delaunay triangulation, there is a dual graph, called the Voronoi diagram. Voronoi diagrams (and thus Delaunay triangulations) arewell-suited for solving closest point problems on a given point set X. Given a set of 2D points, the Voronoi region for a point $\mathrm{Pi}$ is defined as the set of all the points that are closer to $\mathrm{Pi}$ than to any other points. More formally we can say: Let $S=\left\{P_{1}, P_{2}, \ldots, P_{n}\right\}$ be a finite subset of $R^{\mathrm{m}}$ and let $d: \mathrm{R}^{\mathrm{m}} \times \mathrm{R}^{\mathrm{m}} \rightarrow \mathrm{R}$ be $\mathrm{a}$ metric. We define the Voronoi region $\mathrm{VR}\left(\mathrm{P}_{\mathrm{i}}\right)$ of a point $P i$ via $\operatorname{VR}\left(P_{i}\right)=\left\{P \in R^{m} \mid d\left(P, P_{i}\right) \leq d\left(P, P_{j}\right)\right.$ for all $j=1,2, \ldots, n, j \neq i\}$, i.e., $\operatorname{VR}\left(P_{i}\right)$ is the set of all points that are at least as close to $\mathrm{P}_{\mathrm{i}}$ as to any other point of $\mathrm{S}$. The set of all $\mathrm{n}$ VR is called the Voronoi diagram VD (S) of S. [5].

The dual tessellation of Voronoi diagram is known as Delaunay triangulations. The duality comes about in the following way: vertices in the Voronoi diagram correspond to faces in Delaunay triangulation, while Voronoi cells correspond with vertices of Delaunay triangulation. Once the Voronoi diagram for a set of points is constructed, it is a simple matter to produce Delaunay triangulation by connecting any two sites whose Voronoi polygons share an edge. More specifically: let $\mathrm{P}$ be a circle free set. Three points, $p, q$ and $r$ of $P$ define a Delaunay triangle if there is no further point of $\mathrm{P}$ in the interior of the circle which is circumscribed to the triangle $p, q, r$ and its centre lies on a Voronoi vertex. See Fig. 2 for illustration.

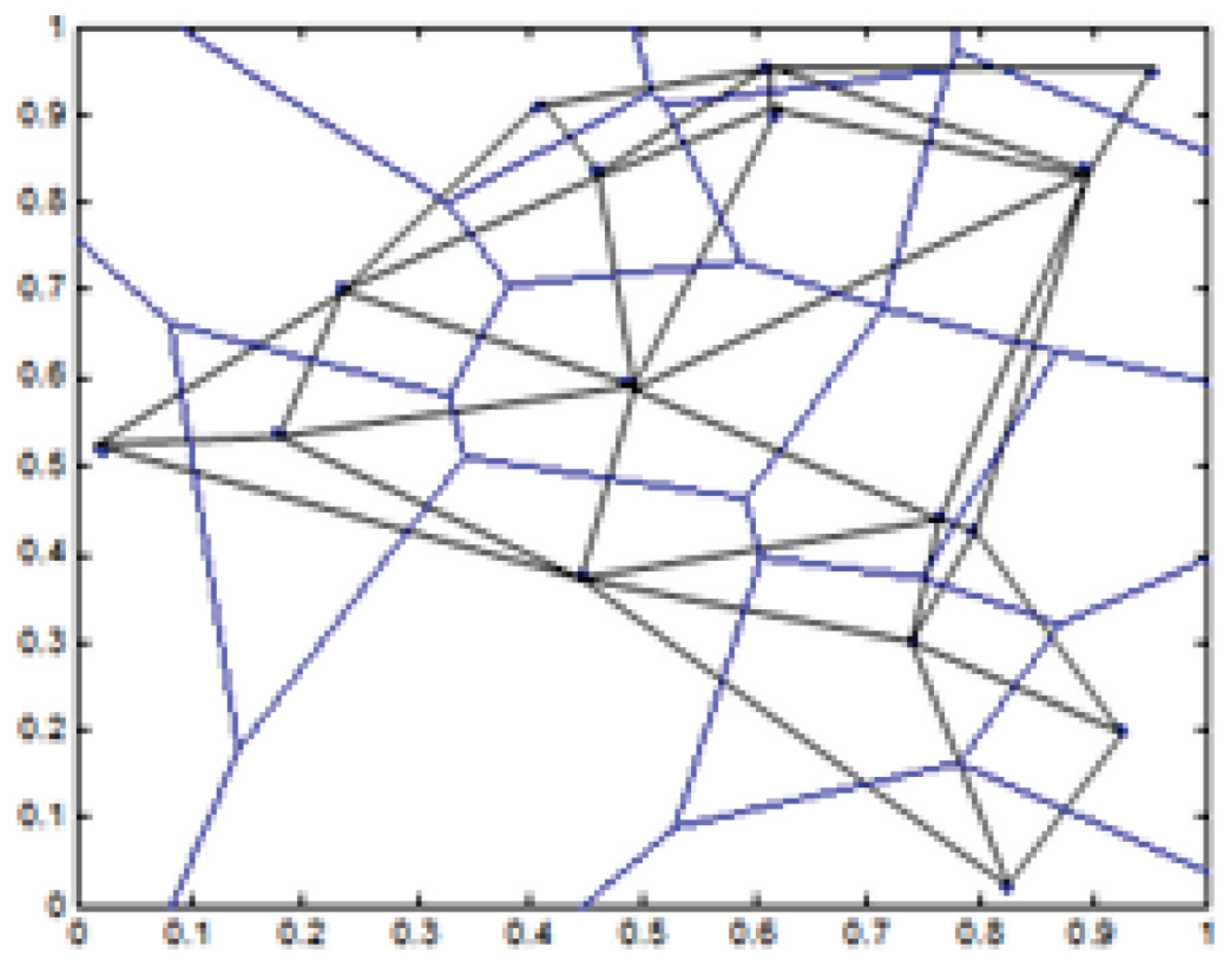

Figure 4:Voronoi Diagram for a Set of 16 Points (Solid Lines): Delaunay Triangulation (Dashed Lines).

There has been considerable research into the design of algorithms for the construction of Delaunay triangulations. According to [15] and [24], there are basically three different approaches, which all rely on the Delaunay criterion. They are "divide and conquer" algorithm which recursively divide the data points up into pieces before finding a Delaunay triangulation for each piece and then merges these triangulations. The "Iteratively build algorithm" which starts with one triangle (with vertices in $\mathrm{X}$ ) and add one point from $\mathrm{X}$ at a time, making sure that at each step every convex quadrilateral in the current triangulation is 
optimally triangulated. The "Post Optimize algorithm" which is very similar to the iteratively build algorithm as it is equally iterative in process.

\section{Applications of Delaunay Triangulation}

A major application of triangulations is the interpolation of functions of two variables, where the function is initially defined only at an irregular set of locations. These locations are used as the vertices of a triangulation. The value of the function at a point, other than a vertex, is computed by performing an interpolation within the triangle containing the point. A triangulation composed of nearly equiangular triangles is considered good for this purpose. [17] and [13] have used the Delaunay triangulation for this purpose.

Another application is the decomposition of polygons into convex sets. An algorithm for decomposing polygons into triangles may be based upon the concept of the Delaunay triangulation. There are applications in pattern recognition and computer graphics for which one wants to combine adjacent triangles into larger convex sets. An $\mathrm{O}(\mathrm{mN})$ algorithm for decomposing a nonconvex polygon of $\mathrm{N}$ sides and $\mathrm{m}$ reflex angles into convex sets is given by [22]. According to [14], Delaunay triangulation is an excellent choice for the terrain fitting and display problem. The Voronoi and Delaunay tessellations have also been extensively used to model spatial patterns in a wide range of fields including astronomy biomathematics, computer science, geography, meteorology, metallurgy, numerical analysis, and packing and covering. The statistical dependence between (Voronoi) neighboring sites may be specified in terms of the Delaunay edge length between sites and the Voronoi border length between their cells [2]. [4] designed a graphical user interface (GUI) which they called DFD (Delaunay Face Detection). This system benefits from the Voronoi properties to detect and segment faces and their features. Their approach is based ultimately on the Voronoi Diagram (VD) technique, which employs a versatile geometric structure that was based on extracting feature points which were then used to define the centers of the Voronoi cells' for image segmentation.

\section{Image Enhancement}

Basically, the idea behind enhancement techniques is to bring out detail that is obscured, or simply to highlight certain features of interest in an image. It is important to keep in mind that enhancement is a very subjective area of image processing. Improvement in quality of degraded images can be achieved by using application of enhancement techniques [19]. Histogram equalization is a reliable technique used for contrast enhancement. The process of enhancing the quality of images from the original image by removing the noise, provide the enhanced image by sharpening the original image and increasing contrast in image [25]. The histogram is a graph showing the number of pixels in an image at each different intensity value found in that image [16]. According to [11], Histogram Equalization (HE) is one of the most popular, computationally fast and simple to implement techniques for contrast enhancement of digital images. [15] said the greater the histogram stretch, the greater the contrast of the image.

\section{Histogram equalization}

Contrast enhancement has great significance in digital image processing. Histogram Equalization (HE) is one of the most popular, computationally fast and simple to implement techniques for contrast enhancement of digital images [11]. A histogram is a graphical representation of the distribution of data. An image histogram is a graphical representation of the number of pixels in an image as a function of their intensity. The histogram equalization technique is used to stretch the histogram of the given image. The Greater the histogram stretch, the greater the contrast of the image [12]. In other words if the contrast of the image is to be increased then it means the histogram distribution of the corresponding image needs to be widened. Histogram equalization is the most widely used enhancement technique in digital image processing because of its simplicity and elegancy. In an image processing context, the histogram of an image normally refers to a histogram of the pixel intensity values. The histogram is a graph showing the number of pixels in an image at each different intensity value found in that image [16]. 


\section{Image Segmentation}

[8] said that image segmentation is a lowlevel image processing task that aims at partitioning an image into homogeneous regions in terms of the features of pixels extracted from the image. The definition of region homogeneity depends on the application. Examples for such homogeneity features are pixel gray level, pixel RGB colour, range of the pixel from the camera, position of the pixel, local covariance matrix [20]. [3] classified image segmentation procedure into boundary representation and regional representation. Each representation is an identification of homogeneous regions or contours of local inhomogeneity, respectively. In the work of [10], Segmentation algorithms for monochrome images generally are based on one of two basic properties of gray-level values: discontinuity and similarity. In the first category, the approach is to partition an image based on abrupt changes in gray level and the principal approaches in the second category are based on thresholding and label region algorithm. According to [21], image segmentation techniques can be classified into three different classes: (1) feature-space based methods which composed of feature extraction and clustering (2) imagedomain based methods which goes through the image and finds the boundary between segments by some rules and (3) edge-based methods which consists of edge detection and edge linking.

\section{Methods}

This system adopts a Rapid Application Development (RAD) method. It is iterative; as one version completes, the next follows. This was adopted because it breaks the entire system into series of versions that are developed sequentially where each requirement is categorized into versions and the most fundamental requirements are bundled into the first version of the system. Our system has some vital underlying functional processes that made it unique. The functions underlying the proposed system benefit from the Voronoi properties to detect and segment images and their features. The first step in every machine vision task is the pre-processing phase, which includes but is and not limited to histogram equalization, mathematicalmorphology, median and low pass filtering. Dual processing is adopted to reduce, as much as possible, the illeffects of any intensive and uneven illumination.

Our first approach is based ultimately on the VD technique, which employs a versatile geometric structure [26][5] and [1]. The approach was based on extracting feature points which were then used to define the centres of the Voronoi cells' for image segmentation. Although our method is in line with them in using Voronoi diagram, our methodology and image targets are completely different. Concise definitions of Voronoi diagram and Delaunay triangulation are presented in section 2.2 above.

Before attempting any operations, we perform a histogram equalization to reduce the lighting ill effect. This is referred to as preprocessing step. Next, the Voronoi diagram is applied. The proposed technique resembles the dynamic thresholding method used for segmentation, but it differs in terms of divide and- merge decision making. Colour images have their colours distributed in such a way that varies significantly from one another. Voronoi diagram is used to construct Delaunay triangulation from points on the image histogram. The outer boundary of Delaunay triangulation is simply the convex hull (CV) of the set of the feature point. Hence, the two global maxima are obtained by extracting the top two values in the Delaunay triangulation list of vertices; which correspond to the peaks in the histogram. The two global maxima are obtained by extracting the top two values in the Delaunay triangulation list of vertices; which correspond to the peaks in the histogram. In order to get the minima that fall between these two peaks, a new set of points are triggered using the following steps:

I. Generate the host image histogram,

ii. Generate Voronoi diagram (VD) /Delaunay triangulation to obtain the list of vertices and get the two peaks,

iii. Set all points below the first peak to zero, and set all points beyond the second peak to zero,

iv. Set all points that are equal to zeros to be equal to the argmax (peak1, peak2)

v. Derive the new points using:

$\mathrm{S}_{\text {new }}(\mathrm{x})=|\mathrm{S}(\mathrm{x})-\max (\mathrm{S}(\mathrm{x}))|$

Where max $(\mathrm{S}(\mathrm{x}))$ denotes the highest frequency in the host image histogram. 
The minima that fall between these two peaks is also extracted and these unique feature points are then sorted in ascending order to outline a one-dimensional vector $(\mathrm{K})$ containing values that form the ranges with which merging and splitting decisions are made. The following process is applied:

Input: Host gray scale image I

Input: Vector generated by the previously described procedure $\mathrm{K}$

Initialize a new vector $\mathrm{L}=[]$

// the vector'd' stores a few gray values that represent our segmented image

Set all pixels in I smaller than $\mathrm{K}(1,1)$ to black (0)
Set all pixels in I greater than $\mathrm{K}($ length $(\mathrm{K}(:)), 1)$ to black (0)

fori $=1$ to length $(\mathrm{K}(:))-1$ do

if $==1$ then

setall $(\mathrm{I}>=\mathrm{V}(\mathrm{i})$ AND I $<=\mathrm{K}(\mathrm{i}+1))$

toK $(\mathrm{i}+1)$

$\mathrm{L}=[\mathrm{L} ; \mathrm{K}(\mathrm{i}+1)]$

else

$\operatorname{setall}(\mathrm{I}>\mathrm{K}(\mathrm{i}) \mathrm{AND} \mathrm{I}<=\mathrm{K}(\mathrm{i}+1))$

toK $(\mathrm{i}+1)$

$\mathrm{L}=[\mathrm{L} ; \mathrm{K}(\mathrm{i}+1)]$

end if

end for

Output: Segmented gray scale image I Segmented

The high level model of the proposed system

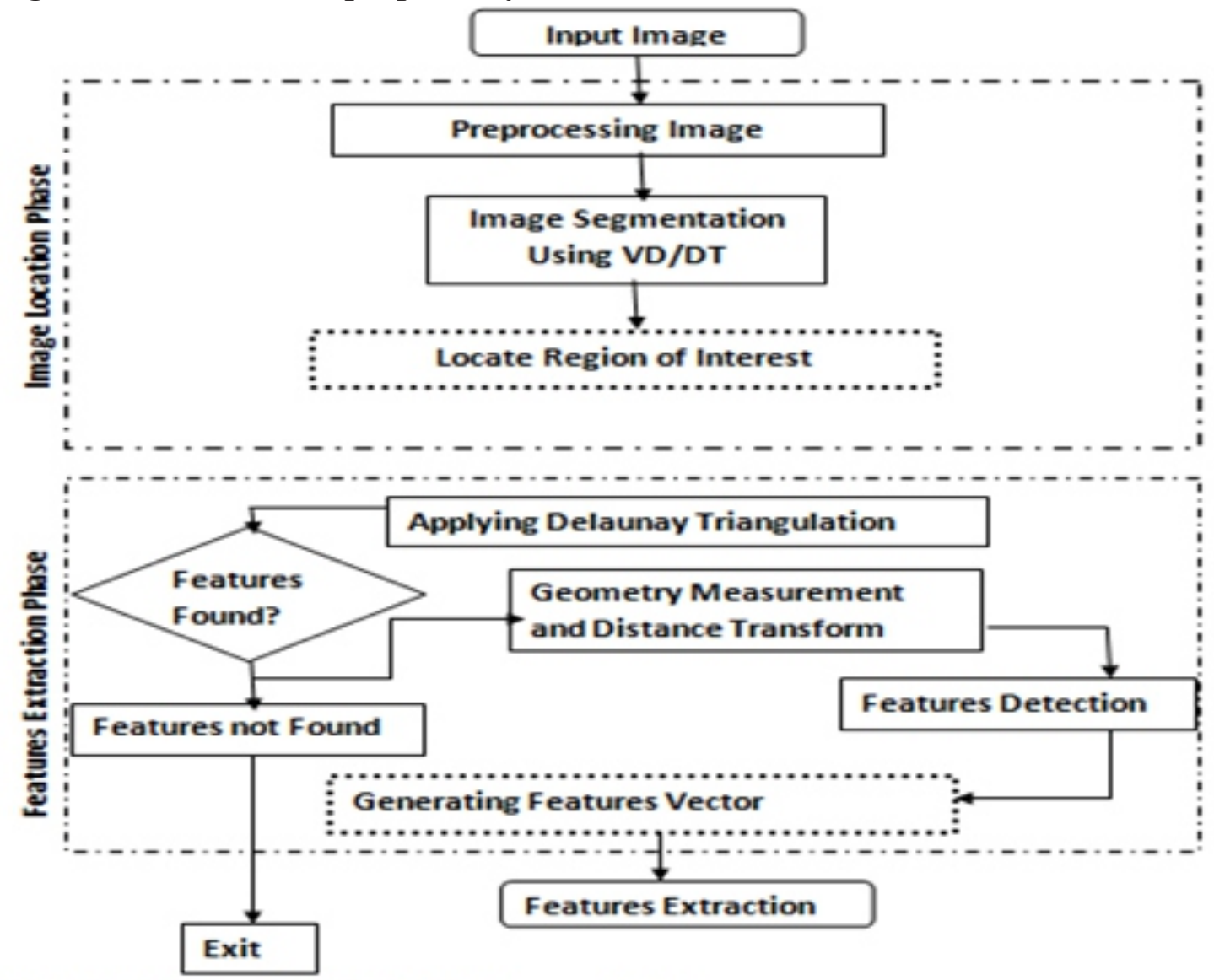

Fig 3: High Level Model of the Proposed System

\section{Data/information gathering}

This research work exploits a number of still digital and radiographic images, few of which are from international recognized databases used for biometric algorithm testing. Others include still images gotten from World Wide Web (www) and of course our own database created for the purpose of this research. Synthetic images were used to create a controlled test environment for the system. This made it possible to validate the algorithm and to illustrate simplified situations that demonstrate challenges provided by the real-world images.

Our experiments were carried out using PC Genuine Intel ${ }^{\circledR}$ CPU T2300 with a $1.67 \mathrm{GHz}$ CPU and $1 \mathrm{~GB}$ of RAM memory. The algorithm was developed using MATLAB (R2010a). Randomly selected samples of images were 
chosen from the various sources and geometric feature base method is deployed to reveal the detected features. The image files extension formats of the selected images are Portable Network Graphics (PNG), Joint Photographic Expert Group (JPEG) and Tagged Image File (TIF).

\section{System Design}

Like every other system, our algorithm comprises of the input design, process design as well as the output design. Fig. 4 represents the input design, Fig. 5, the process design and Fig. 6 stands for the output design.

\section{Input design}

The input data for our algorithm is usually an image which is been imported into the application. Further processes are carried out on the image that changes it into its gray equivalent to remove certain noise and thereafter becomes the input for the histogram equalization process. The Delaunay triangulation process is carried out on the histogram using its peak points after which the features for segmentation are identified.

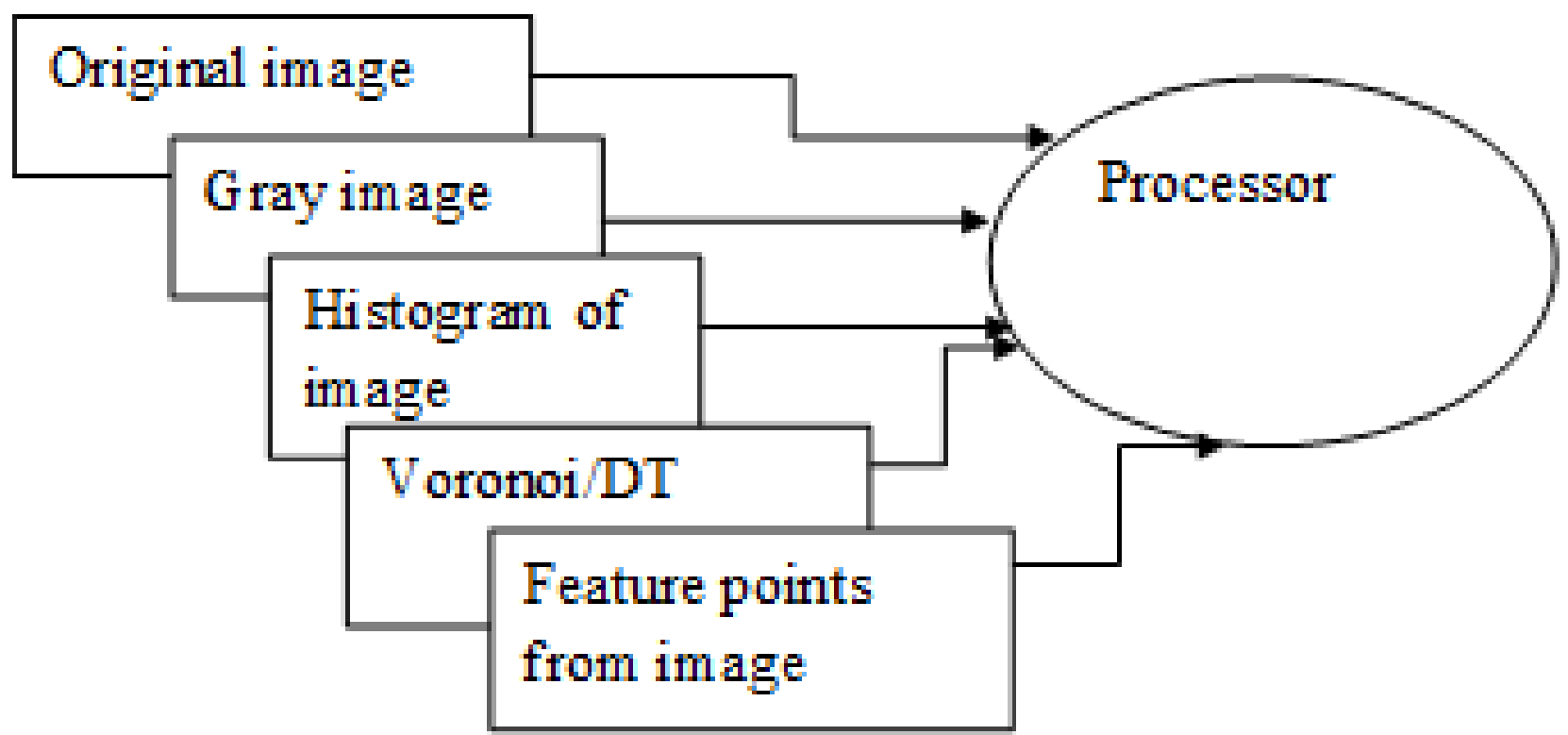

Fig. 4: Input design of a Delaunay triangulation based image segmentation

\section{Algorithm}

Below is a description of the algorithm stating how this can be actualized

(i) Get the image into the work area of the application

(ii) Perform an enhancement on the image

(iii) Change the image to its gray equivalent

(iv) Get a histogram equalization of the image

(v) With the convex hull, perform a Delaunay triangulation

(vi) Indicate the possible iteration

(vii) Begin the iteration

(viii)Check to ensure appropriate iterations are in place

(ix) Produce the segmented image 


\section{System flowchart}

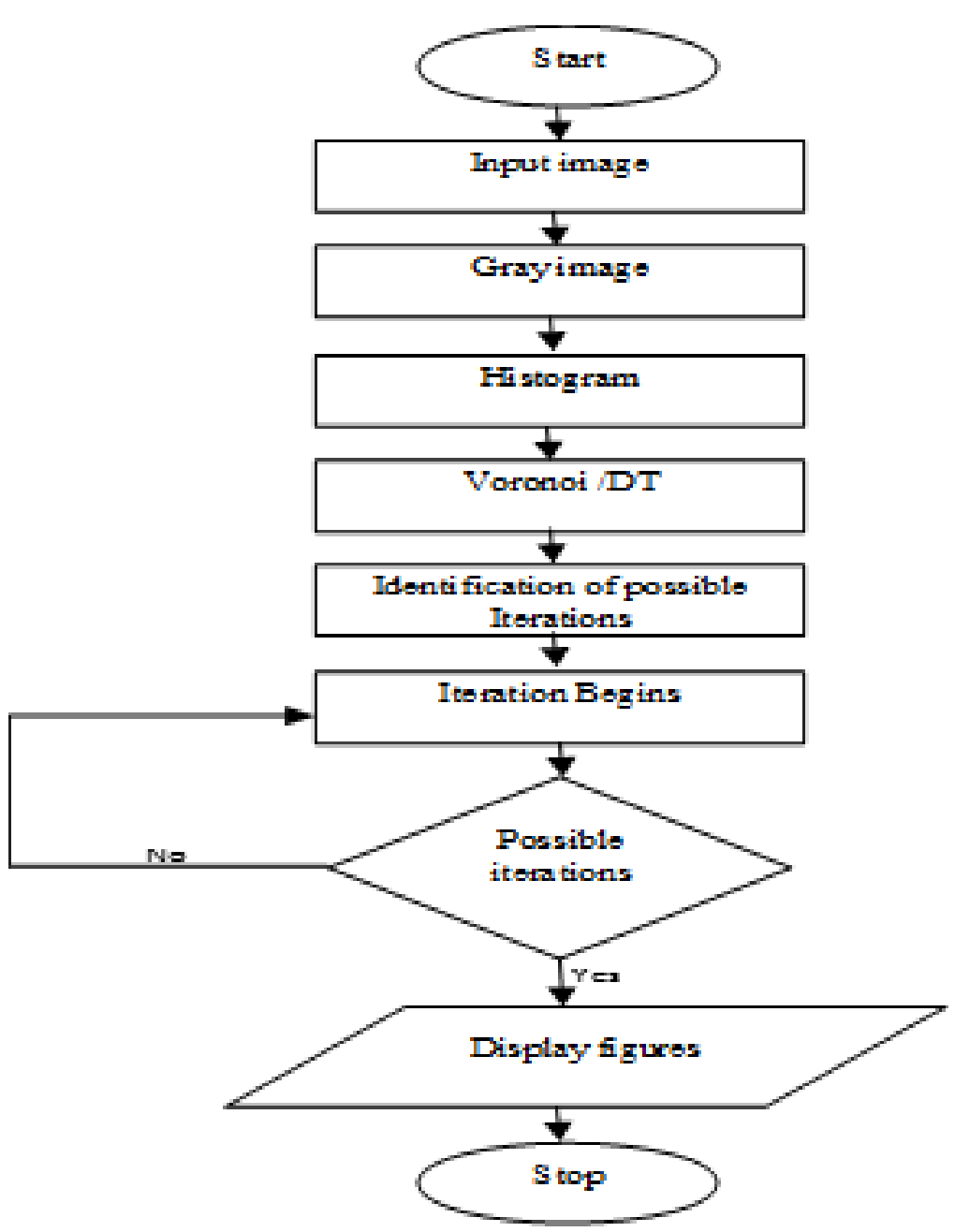

Fig 5: Flow Chart of a DT Based Image Segmentation

\section{Output design}

Our algorithm has the capability of producing expected segmentation as output going by the number of iterations indicated. It is also able to produce the histogram of the equalized image as well as the VD/DT on the image. The original image however is also part of the output to enhance comparison. Figure 9 below illustrates the output design of our system

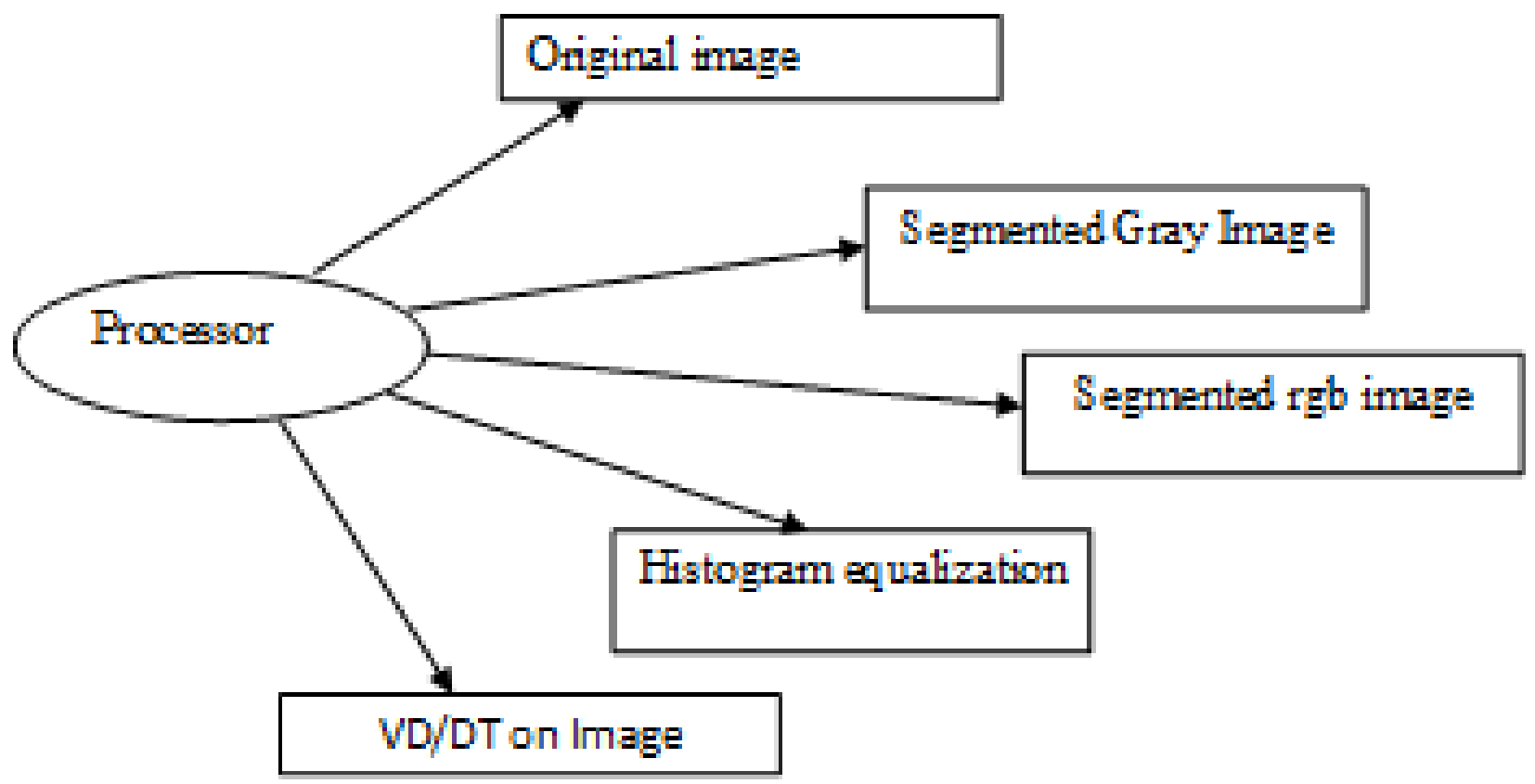

Fig 6: Input Design of a Delaunay Triangulation Based Image Segmentation 


\section{Results and Discussion}

Digital image segmentation using Delaunay Segmentation Algorithm has been implemented on some sampled images and results were obtained. The result therefore shows the breakdown of the processes as well as the relationship that exist among them as shown below.

\section{Implementation}

Our experiments were carried out using PC Genuine Intel(R) CPU T2300 with a
1.67GHz CPU and $1 \mathrm{~GB}$ of RAM memory. The algorithm was developed using MATLAB (R2010a). Randomly selected samples of images were chosen from the various sources and used to test our algorithm. The image files extension formats of the selected images are Portable Network Graphics (PNG), Joint Photographic Expert Group (JPG) and Tagged Image File (TIF). The algorithm produces the same result irrespective of the image file extension format used.

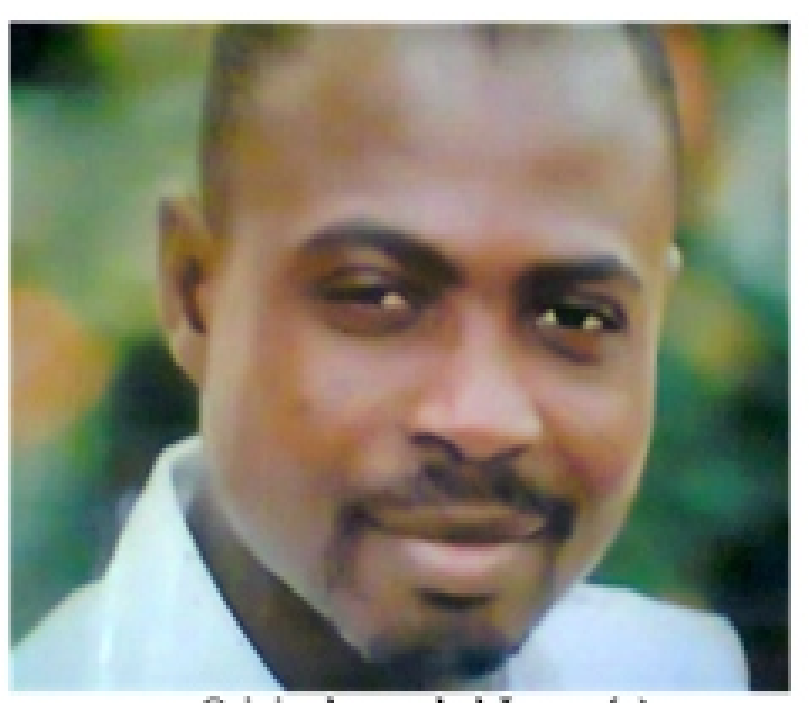

Original ampled Image(a)

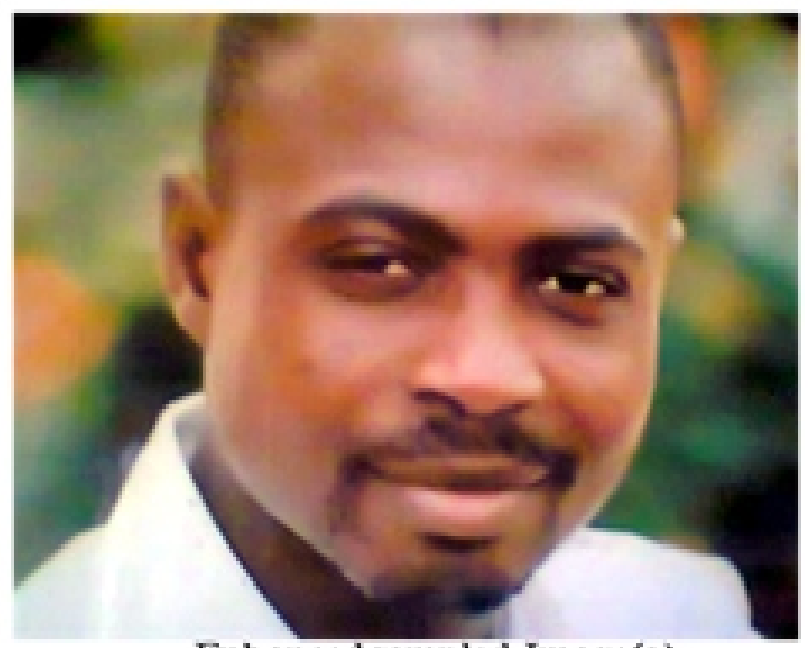

Enhanced sampled Image(a)

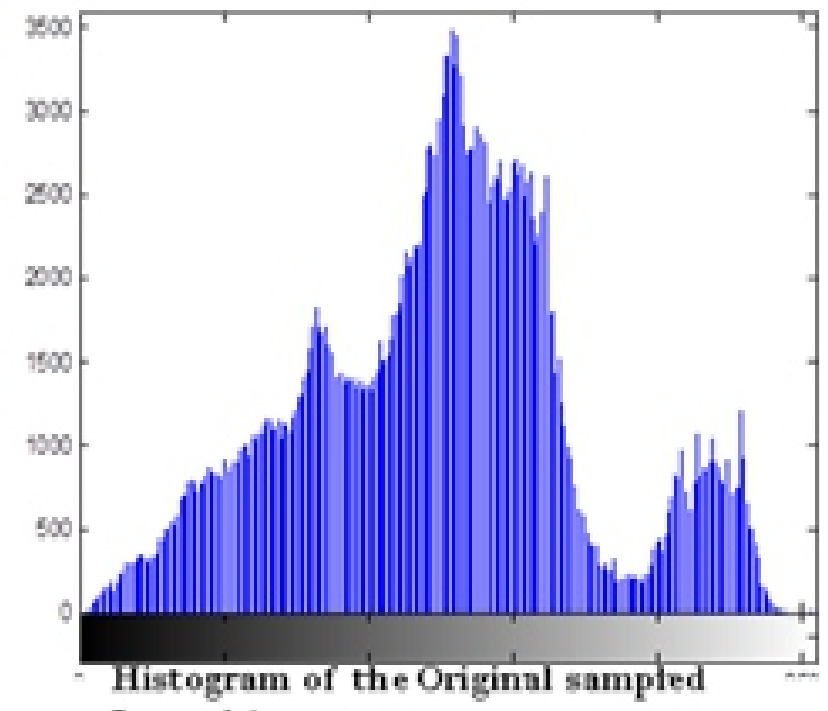

$\operatorname{Image}(\mathrm{a})$

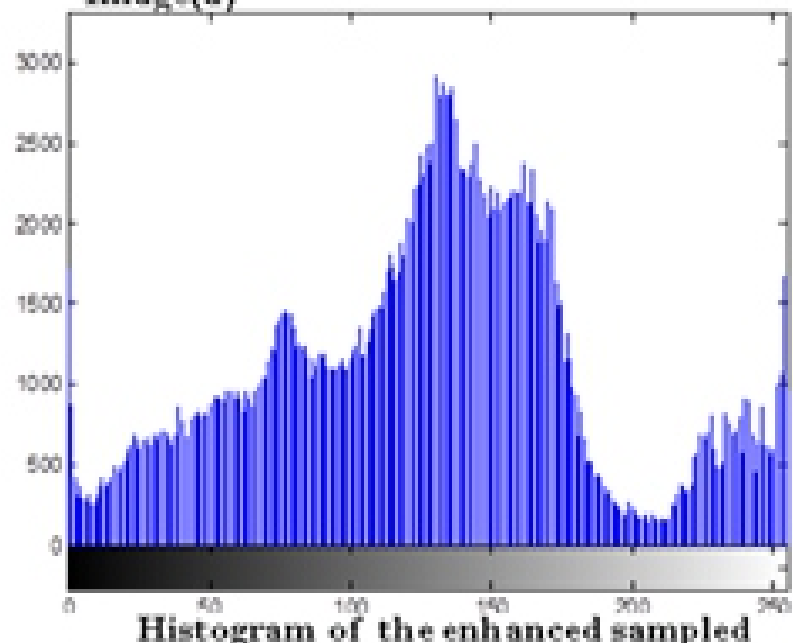

Image(a)

Fig. 7: Display of Original and Enhanced Image (a) and their respective Histograms. 


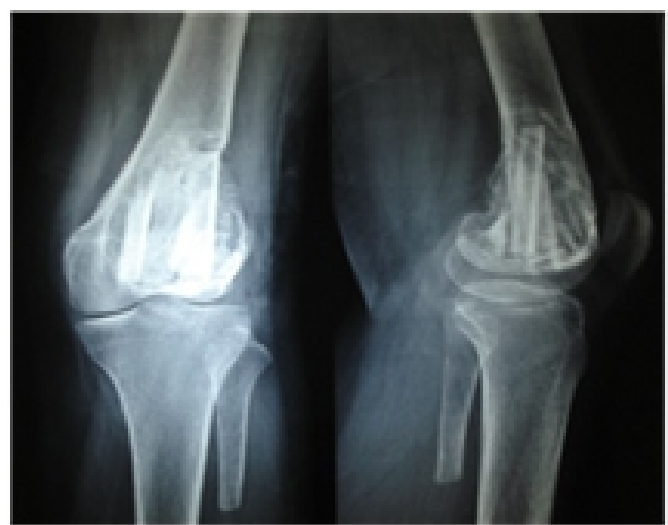

Original sampled Image (b)

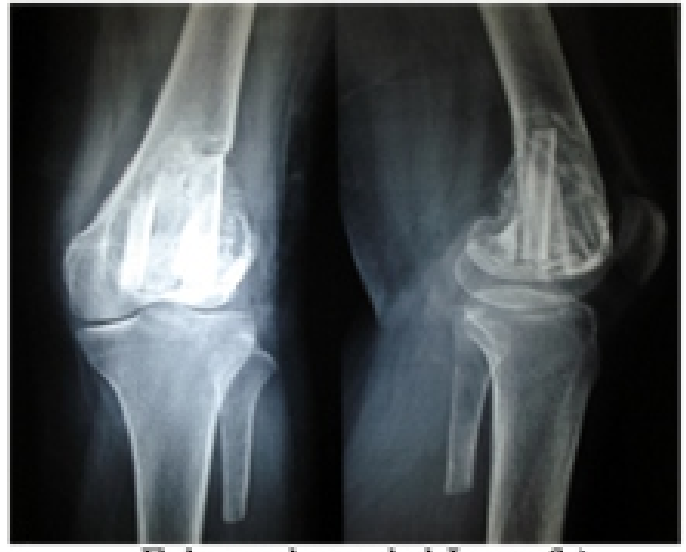

Enh anced sampled Image(b)

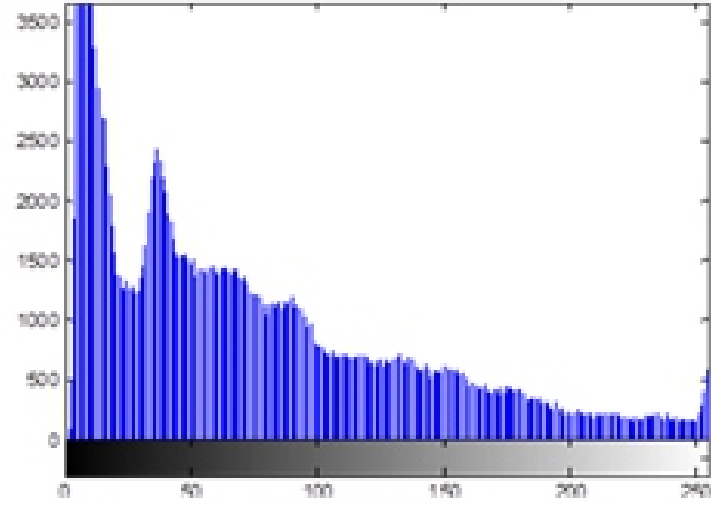

Histogram of the Original sampled Image(b)

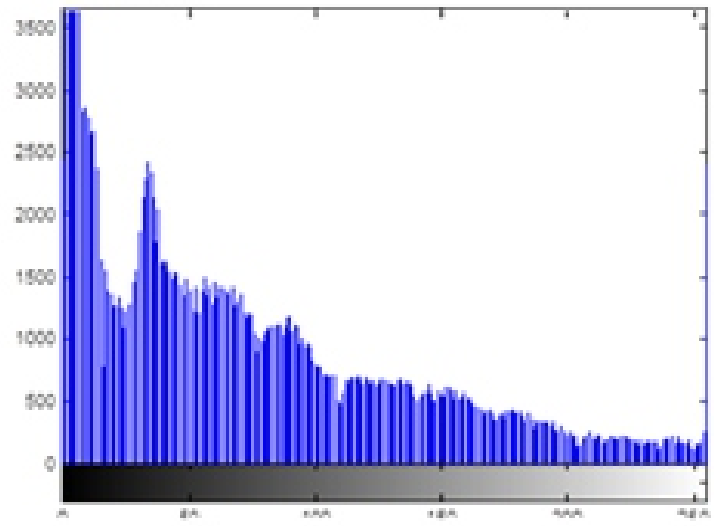

Histogram of the enhanced sampled Im:

Fig 8: Display of Original and Enhanced Images (b) and their respective Histograms.

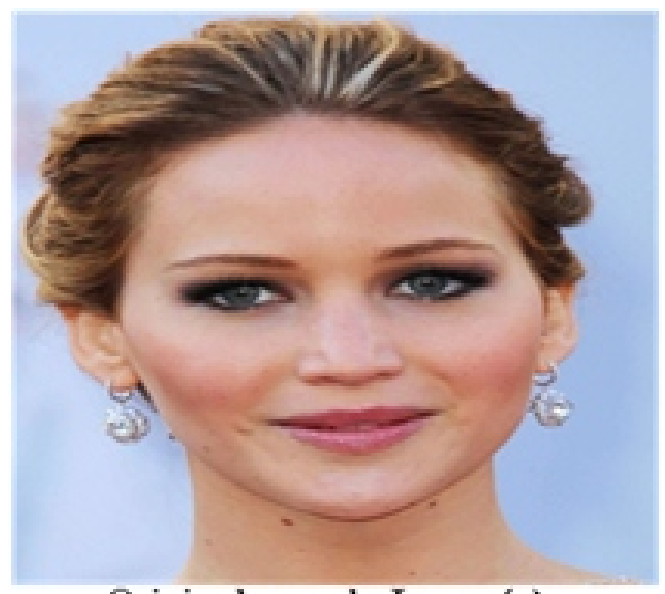

Original sample Image(c)

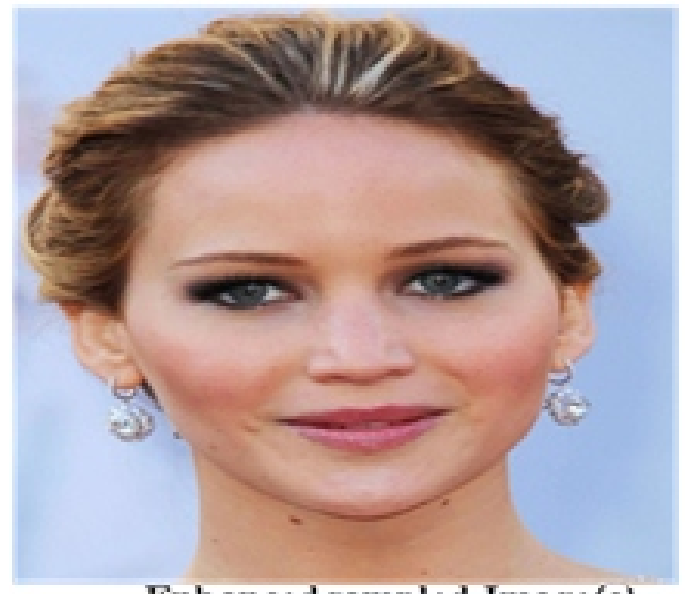

Enh anced sampled Image(c)

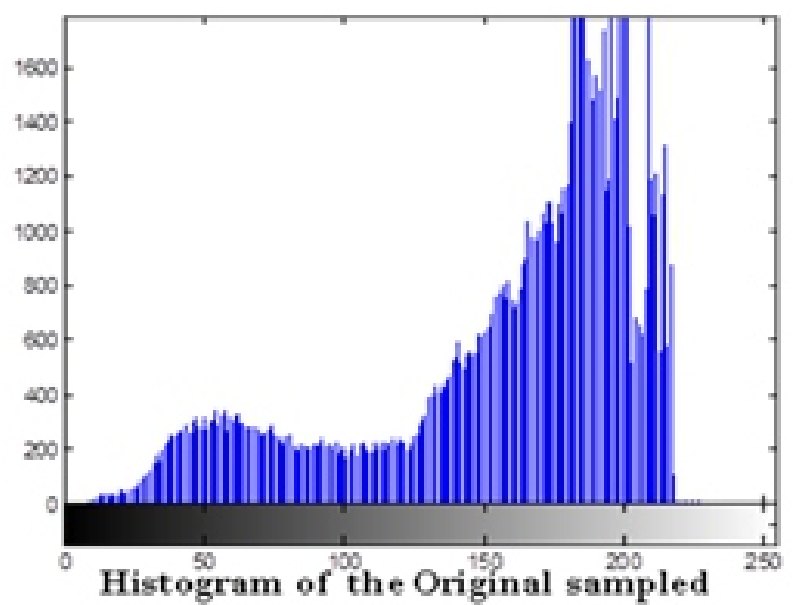

Image(c)

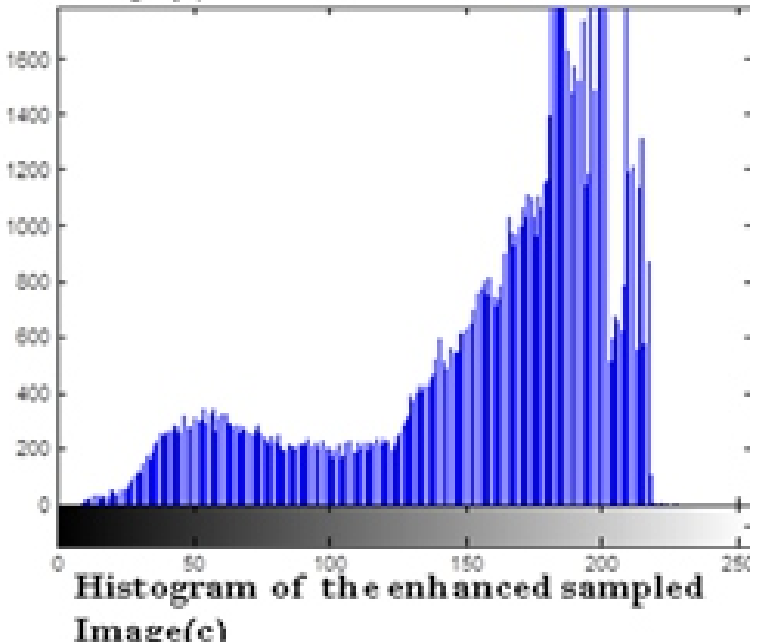

Fig. 9: Display of image (c) before and after enhancement and their respective histograms 


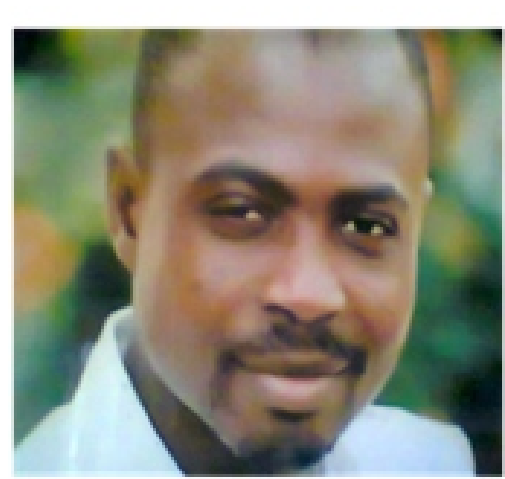

Original Image (a)

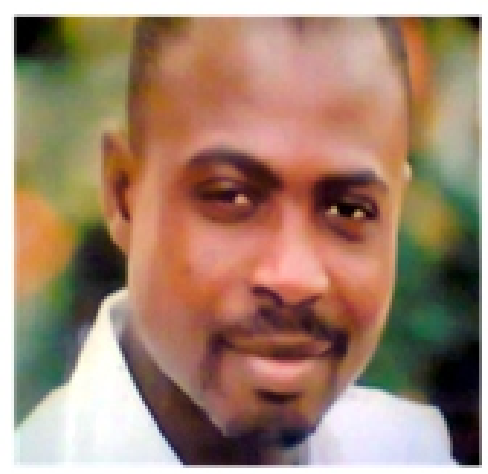

Enh anced Image (a)

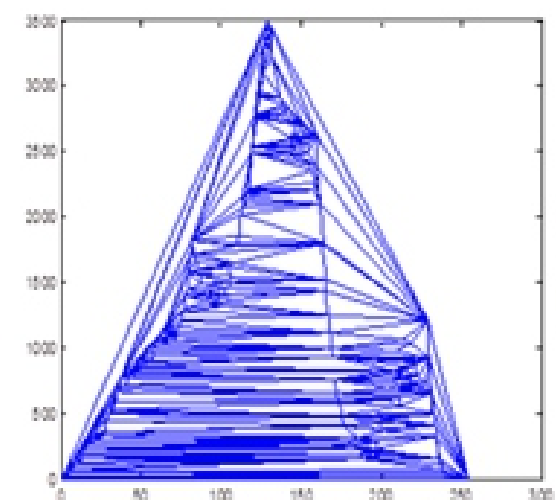

Delaunay triangulation of the histogram of (a)

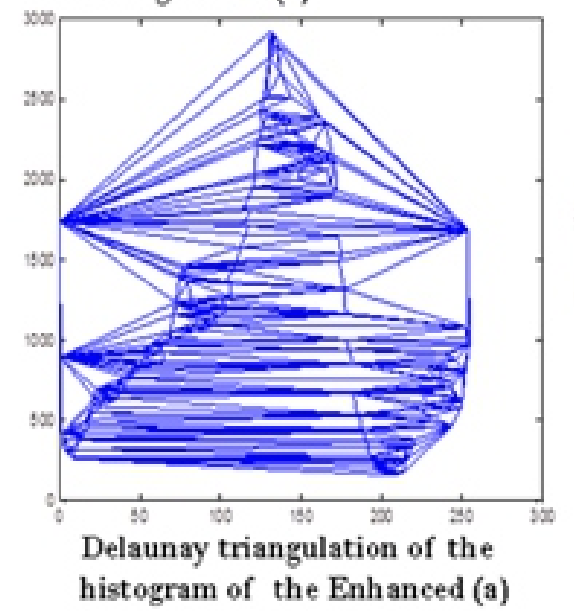

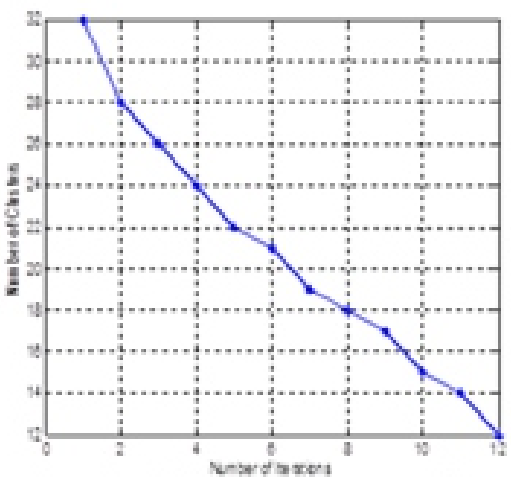

Graph of DT based segmentation of original Image(a)

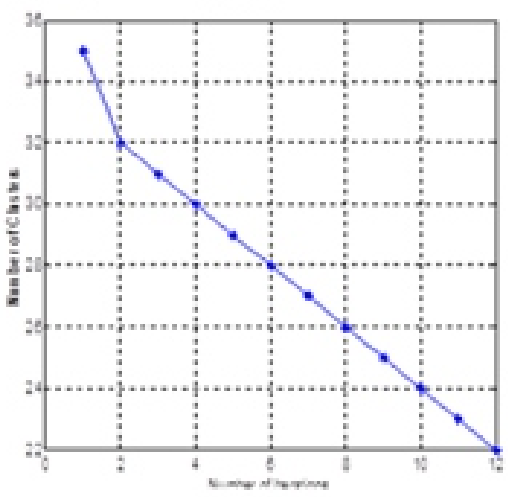

Graph of DT based segmentation of enhanced Image(a)

Fig. 10: Image (a), its respective Delaunay triangulation of the histograms and the graph of the Delaunay based segmentation of the image both for the original image and after enhancement

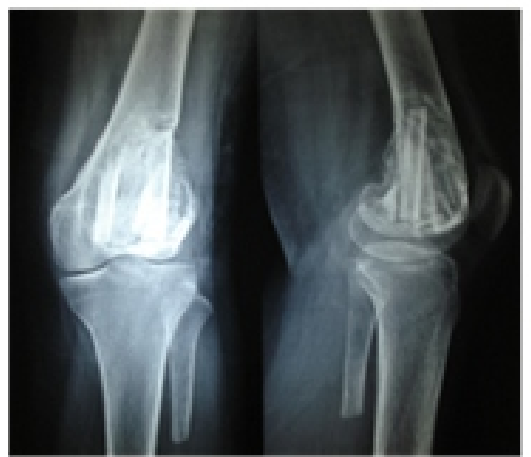

Original Image (b)

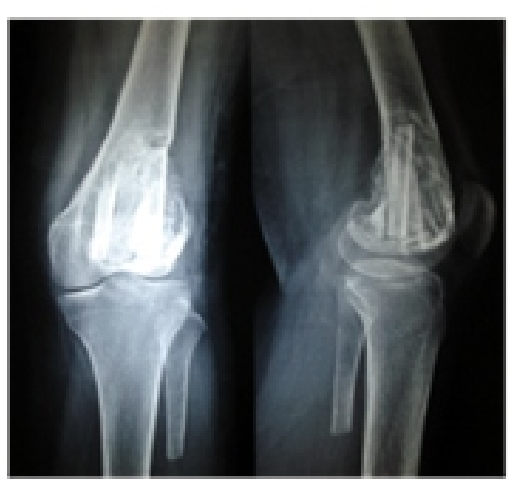

Enhanced Image (b)

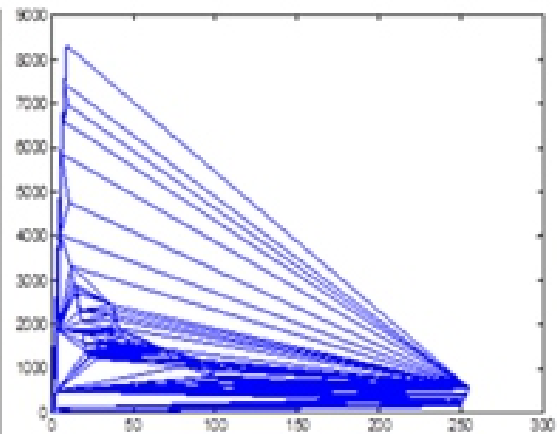

Delaunay triangulation of the histogram of (b)

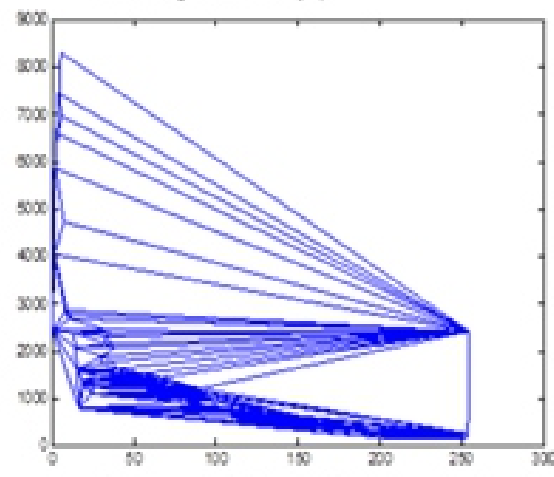

Delaunay triangulation of the histogram of the Enhanced (b)

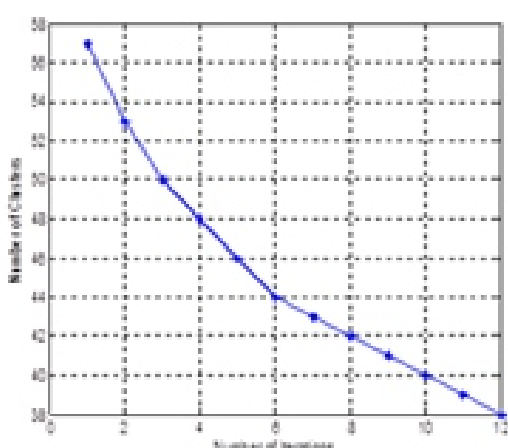

Graph of DT based segmentation of original Image(b)

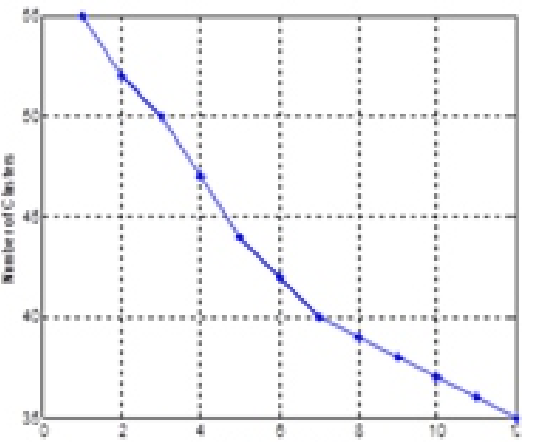

Graph of DT based segmentation of enhanced Image(b)

Fig. 11: Image (b), its respective Delaunay triangulation of the histograms and the graph of the Delaunay based segmentation of the image both for the original image and after enhancement 


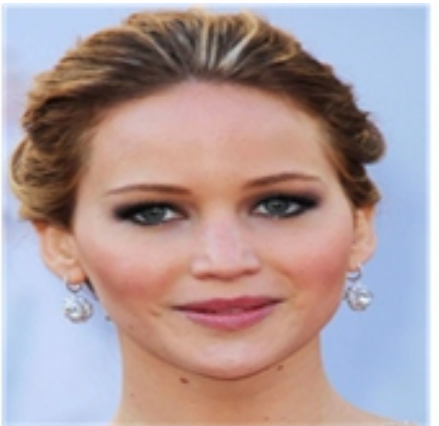

Original sampled Image (c)

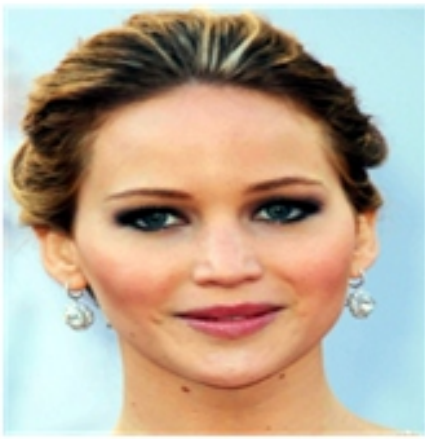

Enhanced sampled Image (c)

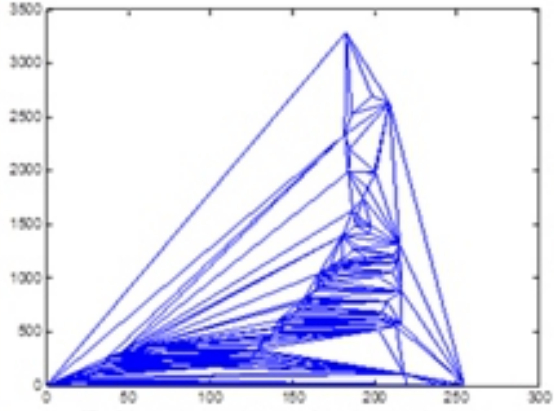

Delaunay triangulation of the histogram of (c)

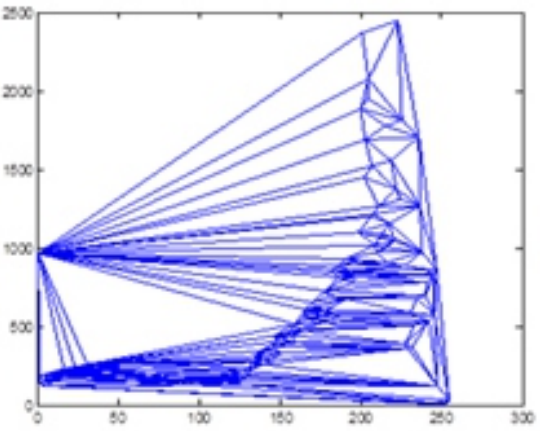

Delaunay triangulation of the histogram of the Enhanced (c)

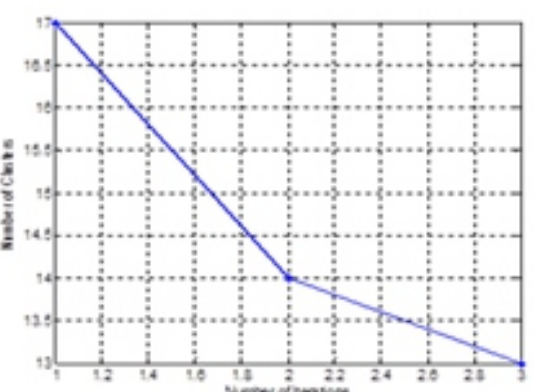

Graph of DT based segmentation of original Image(c)

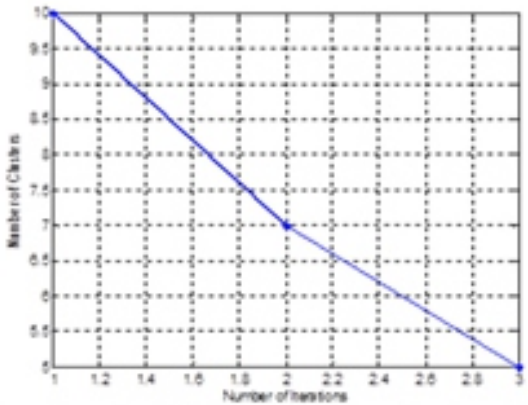

Graph of DT based segmentation of enh anced Image(c)

Fig. 12: Image (a), its respective Delaunay triangulation of the histograms and the graph of the Delaunay based segmentation of the image both for the original image and after enhancement.

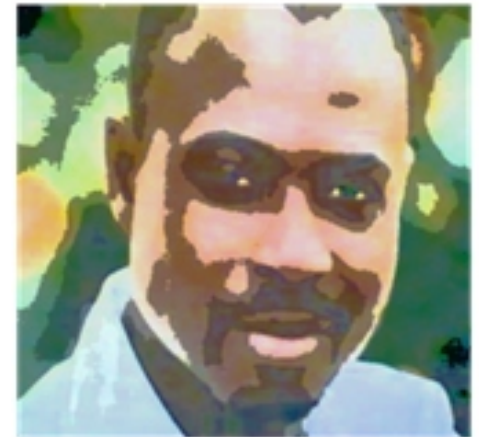

DT based colour segmentation of original Sample Image (a)

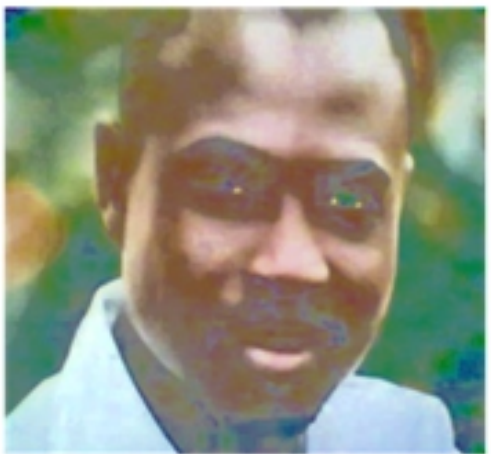

DT based colour segmentation of enhanced Sample Image (a)

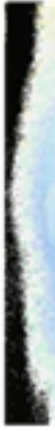

DT based colour segmentation of Original Sample Image (b)

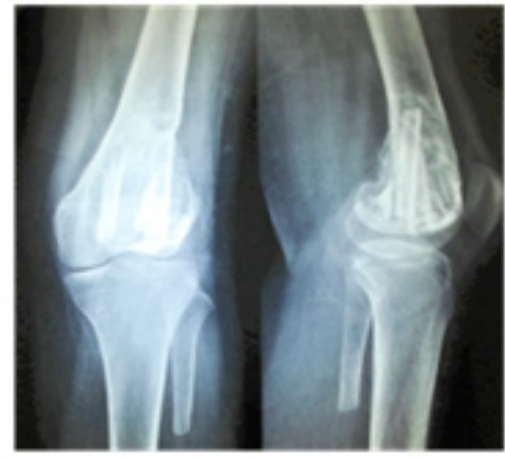

DT based colour segmentation of Enhanced Sample Image (b)

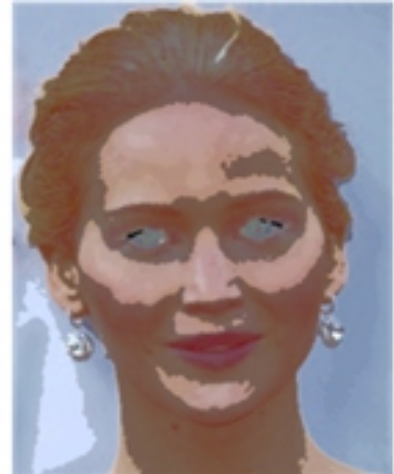

DT based colour segmentation of original Sample Image (c)

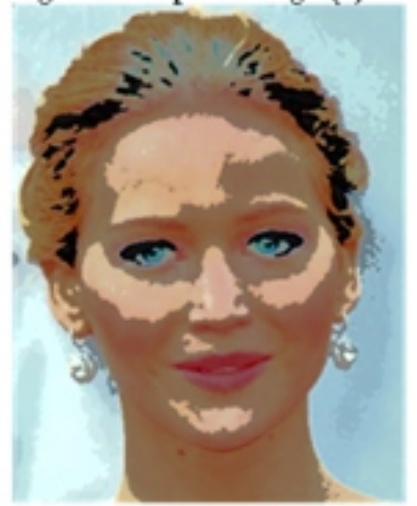

DT based colour segmentation of enhanced Sample Image (c)

Fig. 13: DT based segmentation of images a, b, c for each original and enhanced image. 
Delaunay Segmentation Comparison Chart of the selected images

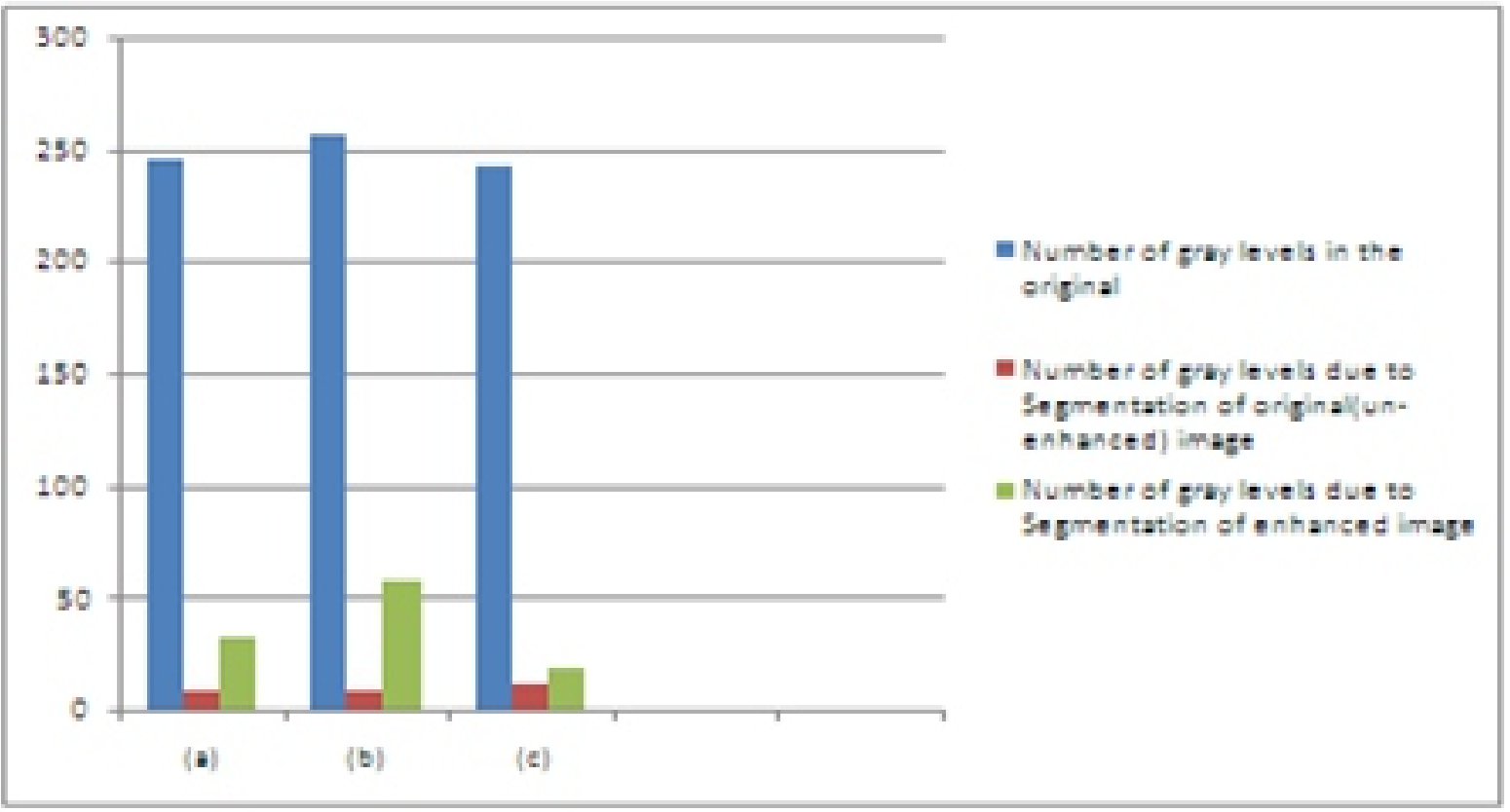

Fig. 14: Chart Showing the Relationship that Exist Between the Number of Gray Values in the Original Images and the Number of Gray Values after DT Segmentation Process Emphasizing the Unequalized and Equalized Techniques.

Table 1: Relationship between Number of Gray value in Original Image, and the Reduced Gray Values Due to Segmentation before and after Enhancement

\begin{tabular}{cccc}
\hline $\begin{array}{l}\text { Sample } \\
\text { Image }\end{array}$ & $\begin{array}{l}\text { Number of gray } \\
\text { levels in the } \\
\text { original }\end{array}$ & $\begin{array}{l}\text { Number of gray levels due to } \\
\text { Segmentation of original(un- } \\
\text { enhanced) image }\end{array}$ & $\begin{array}{l}\text { Number of gray levels due } \\
\text { to Segmentation of } \\
\text { enhanced image }\end{array}$ \\
\hline (a) & 245 & 8 & 32 \\
(b) & 256 & 9 & 58 \\
(c) & $\mathbf{2 4 3}$ & $\mathbf{1 1}$ & $\mathbf{1 8}$ \\
\hline
\end{tabular}

\section{Discussion}

Digital image segmentation using Delaunay Segmentation Algorithm has evolved from several processes as described in our method. The result therefore shows the breakdown of the processes as well as the relationship that exist among them.

Fig. 7, 8 and 9 in our result displays certain category of images labeled sample a, b, c each selected from a collection of digital images. Fig 7,8 and 9 each displays images before and after enhancement and hence their respective histograms. It reveals that for each of the images (a) to (c), there is a display of the original image as exactly captured by the camera or device used and the enhanced form of the image with their respective histogram representations, side by side with the Images. The histogram as seen stretches the images to span from 0 to 255 but its shape is preserved. The histogram of the enhanced images as can be seen in our result gets a flatter response as at when compared with that of the un-enhanced image and this is due to contrast stretching which makes the darkest pixel to be black and the brightest pixel to be white.

Fig. 10,11 and 12 shows the individual images and their respective Delaunay triangulation of the histograms as well as the graph of the Delaunay based segmentation of the images both for the original image and after enhancement. It is observed that the outer boundary of Delaunay triangulation (DT) which is simply the Convex Hull (CV) of the set of the feature points appears to span relatively small gray points which is evident in the number of triangulations obtainable. Clearly, we see that images after undergoing enhancement process are better contrast wise than their original forms as captured by the camera or digital device used. This is evident in the respective graphs showing the effect of numbers of iterations on the clusters generated from a given image. i.e the individual clusters with respect to the number of iterations 
performed on the enhanced form of the sampled images differs in intensity from their respective original images.

Fig. 13 comprises of the DT based colour segmentation of selected Images $\mathrm{a}, \mathrm{b}$ and c showing the resultant segmentations before and after enhancement. We have discovered that a better segmentation is achieved when the image is enhanced since noise is highly reduced as we can see from Fig. 13.

Table 1 features the relationship between number of gray value in original images, and the reduced gray values due to segmentation before and after enhancement. It reveal to us that the gray value number reduction experienced after segmentation is relatively very small in unenhanced images when compared with its value in enhanced images. For an instance, an image sample (b) which has about 256 gray values originally is reduced to 9 and 58 gray values for un-enhanced and enhanced images of (b) respectively.

Figure 19, therefore is a chart representing the relationship that relates the number of gray values of the original image samples (a) to (c) and the values obtained after segmentation. The blue rectangle represents the number of gray levels in the original image, the red stands for the number of gray levels due to segmentation of the original (un-enhanced) image while the green rectangle signifies the number of gray levels obtained due to segmentation of enhanced image. The chart reveals that the segmentation of the enhanced images produces more gray values than the unenhanced image, hence a better segmentation

\section{Conclusion and Recommendations}

Segmentation is an inherently subjective problem and quantitatively measuring performance of different segmentation algorithms is extremely tricky since there is no real "correct" answer to be compared with. Each algorithm has its own relevance as to the kind of result expected. Our algorithm successfully extracts cogent features from a given image and made use of the Delaunay Triangulation to carry out segmentation. This is done by segmenting the image regions into connected homogeneous regions based on their gray values. For better segmentation, histogram equalization was carried out on the selected images to enhance the image contrast. The algorithm was tested on the given images before and after enhancement and the productions compared. The proposed method is robust, precise and independent of translation, rotation, and scaling. However, the presence of background intensities similar to the intensities in the image may cause problem for segmentation decisions. We believe that our algorithm can be injected into many different scientific fields; for example in medical images to recognize specific regions or parts, in facial image detection for criminal investigations at police stations, in surveillance tasks, in dictating specific points in satellite images as well as captured submarine images. We therefore recommend that in order to proffer solution to the increasing demand of medical imaging in the diagnosis and treatment of diseases, proper segmentation should be carried out on the image(s) so as to clearly identify points of interest. Also to enhance clarity in viewing scenes of nature e.g. the sky, water bodies, satellite images, crop yield and defect as well as colour disparity check, good digital segmentation technique should be employed.

\section{References}

Ahuja, N. (1982). Dot Pattern Processing Using Voronoi Neighborhoods. IEEE Trans on Pattern Recognition and Machine Intelligence, 4(3):336-343.

Besag,J. (1974). Spatial Interaction and the Statistical Analysis of Lattice Systems. Journal of Royal Statical Society, B 36(2):192-236.

Bowyer, K. and Ahuja, N. (1996). Advances in Image Understanding. Los Alamitos, CA: IEEE Computer Society Press. Pp. 280-300.

Cheddad, A., Mohamad, D. and AbdManaf, A. (2008). Exploiting Voronoi diagram Properties in Face Segmentation and Features Extraction. Pattern Recognition, 41(12):3842-3859.

Costa L. and Cesar, R. (2001). Shape Analysis and Classification. USA: CRC Press. 680pp.

Delaunay, B. (1934) Sur la sphere vide. Bulletin of Academy of Sciences of the USSR, 1(1) 793-800.

Deng Y. and Manjunath B. S. (2001). Unsupervised Segmentation of ColourTexture Regions in Images and Video. IEEE Trans. Pattern Analysis Machine 
Intell, 23(8): 800-810.

Duda, R. O. and Hart, P. E. (1973). Pattern Classification and Scene Analysis. NewYork: John Wiley \& Sons. 512pp.

Gerrits, D., Gabriels, R. and Kooijmans, P. (2006). A Survey of Mesh Generation Techniques, Lecture Notes on Advanced algorithms, Technical University Eindhoven.

Gonzalez, R. C. and Woods, R. E. (1992). Digital Image Processing. USA: Addison-Wesley Publishing Company. Pp 7-9, 413-414.

Hum, Y. C., Lai, K. W., Mohamad-Salim, M. I. (2014). MultiobjectivesBihistogram Equalization for Image Contrast Enhancement.ISI Indexed Journal, 20 (2):22-36.

Laughlin, S. B (1981). A simple coding procedure enhances a neuron's information capacity. Z. Naturforsch, 36(36):910-912.

Lawson, C. L. (1977). Software for Surface Interpolation. In:Rice J.(eds). Mathematical Software III. New York: Academic Press. Pp 161-194.

Lee, D. T. (1977). Proximity and Reachabitity in the Plane. Ph.D. Thesis, Coordinated Science Laboratory Report ACT-12, University of Illinois, Urbana, Illinois, New York: c Press.

Lewis, B. A. and Robinson, J. S. (1978). Triangulation of Planar Regions with Applications. The Computer Journal, 21(4):324-332.

Mandeep K., Kiran J. and Virender L.(2013). Study of Image Enhancement Techniques: A Review. International Journal of Advanced Research in Computer Science and Software Engineering, 3(4):846-848.

McLain, D. H. (1976). Two Dimensional Interpolation from Random Data. The
Computer Journal, 19(2):178-181.

Preparata, F. P. andShamos, M.I. (1988). Computational Geometry. New York: Springer. 398pp

Ramos, V. and Muge, F. (2000). Image Colour Segmentation by Genetic Algorithms. Lisboa-Portugal: CVRM.Pp 1-5.

Rajesh, G., Bhawna, M. and Sheetal, G. (2011). Histogram Equalization Techniques for Image Enhancement. International Journal of Advanced Research in Computer Science and Software Engineering, 2(1):107-111

Satish, K. and Raghavendra S. (2013). A Study on Image Segmentation and its Methods. International Journal of Advanced Research in Computer Science and Software Engineering, 3(9):1112-1114.

Schachter, B., Rosenfeld, A. and Davis, L. S. (1978). Random Mosaic Models for Textures. IEEE Transaction on Systems, Man, and Cybernetics, 8(9):694-702.

Schumaker, L. L. (1993) Triangulations in CAGD. IEEE Computer Graphics and Applications, 13(1):47-52.

Shamos, M. I. andHoey, D. (1975). ClosestPoint Problems. Proceedings of the 16th Annual Symposium on the Foundations of Computer Science,Pp 151-162.

Sumithra, K., Buvana, S. and Somasundaram, R. (2015). A Survey on Various Types of Image Processing Technique. International Journal of Engineering Research \& Technology (IJERT), 4(3):399-403.

Xiao, Y. and Yan, H. (2002). Facial Feature Location with Dela unay Triangulation/Voronoi Diagram Calculation.Proceeding of the 2001 PanSydney Area Workshop on Visual Information Processing(VIP2001).Pp 103-108. 